(c) 2006 International Press

Adv. Theor. Math. Phys. 10 (2006) 747-757

\title{
Oriented matroid theory as a mathematical framework for $M$-theory
}

\author{
J.A. Nieto \\ Facultad de Ciencias Físico-Matemáticas de la Universidad Autónoma de \\ Sinaloa, 80010 Culiacán Sinaloa, Mexico \\ nieto@uas.uasnet.mx

\begin{abstract}
We claim that $M$ (atroid) theory may provide a mathematical framework for an underlying description of $M$-theory. Duality is the key symmetry which motivates our proposal. The definition of an oriented matroid in terms of the Farkas property plays a central role in our formalism. We outline how this definition may be carried over $M$-theory. As a consequence of our analysis, we find a new type of action for extended systems which combines dually the $p$-brane and its dual $p^{\perp}$-brane.
\end{abstract}

In the references [1-5] were established a number of connections between oriented matroid theory [6] and several ingredients of $M$-theory [7-9] including $D=11$ supergravity, Chern-Simons theory, string theory and $p$-brane physics. The real motivation for such connections has been to implement a kind of "duality principle" in $M$-theory via oriented matroid theory. As it is

e-print archive: http://lanl.arXiv.org/abs/hep-th/0506106 
known, duality between various superstring theories was the key symmetry to suggest the existence of an underlying $M$-theory which includes the five consistent superstring theories and $D=11$ supergravity. However, in spite of several interesting proposals for describing $M$-theory (see ref. [10] and references therein), the precise connection between duality and $M$-theory as well as the correct definition of $M$-theory remains as a mystery [8]. Our claim in this work is that oriented matroid theory may provide the necessary mathematical tools for considering a general concept of a duality principle and consequently may establish the bases for a definition of $M$-theory as a "duality theory".

Let us start off with the consideration of the oriented matroid concept. There are several equivalent definitions of an oriented matroid, but perhaps for physicists the most convenient one is in terms of the so-called chirotopes (see ref. [6] and references therein). Namely, an oriented matroid $\mathcal{M}$ is a pair $(E, \chi)$, where $E$ is a non-empty finite set and $\chi$ (called chirotope) is a mapping $E^{r} \rightarrow\{-1,0,1\}$, where $r$ means the rank on $E$, satisfying the following properties:

$(\chi 1) \chi$ is not identically zero,

$(\chi 2) \chi$ is alternating,

$(\chi 3)$ for all $x_{1}, x_{2}, \ldots, x_{r}, y_{1}, y_{2}, \ldots, y_{r} \in E$ such that

$$
\chi\left(x_{1}, x_{2}, \ldots, x_{r}\right) \chi\left(y_{1}, y_{2}, \ldots, y_{r}\right) \neq 0,
$$

there exists an $i \in\{1,2, \ldots, r\}$ such that

$$
\begin{aligned}
& \chi\left(y_{i}, x_{2}, \ldots, x_{r}\right) \chi\left(y_{1}, y_{2}, \ldots, y_{i-1}, x_{1}, y_{i+1}, \ldots, y_{r}\right) \\
& \quad=\chi\left(x_{1}, x_{2}, \ldots, x_{r}\right) \chi\left(y_{1}, y_{2}, \ldots, y_{r}\right) .
\end{aligned}
$$

Here, we assume that $x_{1}, x_{2}, \ldots, x_{r} \in E$.

One of the reasons why physicists may become interested in this oriented matroid definition is because alternating objects are very well-known in higher dimensional supergravity and $p$-branes physics (see ref. [11] and references therein). This can be clarified further if we consider the case of a vector configuration in which case the chirotope $\chi$ can be written as

$$
\chi\left(\mu_{1}, \ldots, \mu_{r}\right) \equiv \operatorname{sign} \operatorname{det}\left(b^{\mu_{1}}, \ldots, b^{\mu_{r}}\right) \in\{-1,0,1\}
$$

for all $b^{\mu_{1}}, \ldots, b^{\mu_{r}} \in R^{r}$ and for all $\mu_{1}, \ldots, \mu_{r} \in E$. In this case, expressions (1) and (2) become connected with the Grassmann-Plücker relations (see ref. [6], Section 3.5). It turns out that expression (3) can also be written in 
the alternative way

$$
\chi\left(\mu_{1}, \ldots, \mu_{r}\right) \equiv \operatorname{sign} \Sigma^{\mu_{1} \cdots \mu_{r}},
$$

where

$$
\Sigma^{\mu_{1} \cdots \mu_{r}} \equiv \varepsilon^{a_{1} \cdots a_{r}} b_{a_{1}}^{\mu_{1}} \cdots b_{a_{r}}^{\mu_{r}} .
$$

Here, $\varepsilon^{a_{1} \cdots a_{r}}$ is the completely antisymmetric symbol. Thus, if we introduce the base $\omega_{\mu_{1}}, \omega_{\mu_{2}}, \ldots, \omega_{\mu_{r}}$ one discovers that (5) leads to the $r$-form

$$
\boldsymbol{\Sigma}=\frac{1}{r !} \Sigma^{\mu_{1} \cdots \mu_{r}} \omega_{\mu_{1}} \wedge \omega_{\mu_{2}} \wedge \cdots \wedge \omega_{\mu_{r}},
$$

which can also be written as

$$
\boldsymbol{\Sigma}=\mathbf{b}_{1} \wedge \mathbf{b}_{2} \wedge \cdots \wedge \mathbf{b}_{r}
$$

Therefore, $\boldsymbol{\Sigma}$ can be identified as a decomposable $r$-form element of $\wedge_{r} R^{n}$. Here, $\wedge_{r} R^{n}$ denotes a $\left(\begin{array}{l}n \\ r\end{array}\right)$-dimensional real vector space of alternating $r$-forms on $R^{n}$.

In ref. [1] it was shown that a local version of (5),

$$
\Sigma^{\mu_{1} \cdots \mu_{r}} \longrightarrow \sigma^{\mu_{1} \cdots \mu_{p+1}}(\xi),
$$

with $r=p+1$, suggested by the so-called matroid bundle [12-15], allows to establish a connection between matroids and Schild type action for $p$-branes [16] (see also ref. [17]),

$$
S_{p}=\frac{1}{2} \int d^{p+1} \xi\left(\gamma^{-1} \sigma^{\mu_{1} \cdots \mu_{p+1}} \sigma_{\mu_{1} \cdots \mu_{p+1}}-\gamma T_{p}^{2}\right),
$$

where $T_{p}$ is a fundamental constant measuring the inertia of the $p$-brane, $\gamma$ is a Lagrange multiplier and

$$
\sigma^{\mu_{1} \cdots \mu_{p+1}}=\varepsilon^{a_{1} \cdots a_{p+1}} v_{a_{1}}^{\mu_{1}}(\xi) \cdots v_{a_{p+1}}^{\mu_{p+1}}(\xi)
$$

with

$$
v_{a}^{\mu}(\xi)=\partial_{a} x^{\mu}(\xi),
$$

where $x^{\mu}(\xi)$ are $d+1$-scalar fields (see ref. [1] for details). One of the interesting aspects of this connection is that duality becomes part of the $p$-brane structure in a systematic way. In order to clarify this observation, let us recall the definition of the dual oriented matroid $\mathcal{M}^{*}$. It turns out that there are different equivalent forms for introducing duality in oriented matroid theory. In terms of chirotopes the dual oriented matroid $\mathcal{M}^{*}$ is defined as 
follows. First, one introduces the dual chirotope $\chi^{*}$ such that

$$
\chi^{*}: E^{n-r} \longrightarrow\{-1,0,1\}
$$

and

$$
\left(x_{1}, x_{2}, \ldots, x_{n-r}\right) \longrightarrow \chi\left(x_{1}^{\prime}, x_{2}^{\prime}, \ldots, x_{r}^{\prime}\right) \operatorname{sign}\left(x_{1}, x_{2}, \ldots, x_{n-r}, x_{1}^{\prime}, x_{2}^{\prime}, \ldots, x_{r}^{\prime}\right),
$$

where $\left(x_{1}^{\prime}, x_{2}^{\prime}, \ldots, x_{r}^{\prime}\right)$ means some permutation of $E \backslash\left(x_{1}, x_{2}, \ldots, x_{n-r}\right)$ and

$$
\operatorname{sign}\left(x_{1}, x_{2}, \ldots, x_{n-r}, x_{1}^{\prime}, x_{2}^{\prime}, \ldots, x_{r}^{\prime}\right)
$$

is the parity of the number of inversions of $(1,2, \ldots, n)$. It is not difficult to see that, as in the case of ordinary matroids [18], every oriented matroid $\mathcal{M}(E, \chi)$ has an associated unique dual $\mathcal{M}^{*}\left(E, \chi^{*}\right)$. Furthermore, it is found that $\mathcal{M}^{* *}=\mathcal{M}$ (see ref. [6], Section 3.4). Assuming that similar duality result should go over a matroid bundle scenario, one finds that the object $\sigma^{\mu_{1} \cdots \mu_{p+1}}(\xi)$ associated with the chirotope $\chi$ of $\mathcal{M}(\xi)$ should imply an identification of the dual of $\sigma^{\mu_{1} \cdots \mu_{p+1}}(\xi)$ in the form

$$
{ }^{*} \sigma^{\mu_{p+2} \cdots \mu_{d+1}}=\frac{1}{(p+1) !} \varepsilon_{\mu_{1} \cdots \mu_{p+1}}^{\mu_{p+2} \cdots \mu_{d+1}} \sigma^{\mu_{1} \cdots \mu_{p+1}},
$$

where the dual ${ }^{*} \sigma^{\mu_{p+2} \cdots \mu_{d+1}}$ is associated with the chirotope $\chi^{*}$ of the dual oriented matroid $\mathcal{M}^{*}(\xi)$. Here, ${ }^{*} \sigma^{\mu_{p+2} \cdots \mu_{d+1}}$ is given by

$$
{ }^{*} \sigma^{\mu_{p+2} \cdots \mu_{d+1}}=\varepsilon^{\hat{a}_{p+2} \cdots \hat{a}_{d+1}} v_{\hat{a}_{p+2}}^{\mu_{p+2}}(\xi) \cdots v_{\hat{a}_{d+1}}^{\mu_{d+1}}(\xi),
$$

where

$$
v_{\hat{a}}^{\mu}(\xi)=\partial_{\hat{a}} x^{\mu}(\xi) .
$$

In order for (16) and (17) to make sense, it is necessary to consider that locally the fiber bundle $\mathcal{E}(B, F, G)$, with base space $B$, fiber $F$ and structural group $G$, is parametrized by coordinates $\xi=\left(\xi^{a}, \xi^{\hat{a}}\right)$. Moreover, the variables $v_{a}^{\mu}(\xi)$ and $v_{\hat{a}}^{\mu}(\xi)$ should be associated with the horizontal part $H_{\xi}(\mathcal{E})$ and the vertical part $V_{\xi}(\mathcal{E})$ of the tangent bundle $T_{\xi}(\mathcal{E})$, respectively. Of course, we have that $v_{a}^{\mu}(\xi)$ and $v_{\hat{a}}^{\mu}(\xi)$ satisfy the orthogonality condition

$$
v_{\hat{a}}^{\mu} v_{a \mu}=0 .
$$

From the point of view of ordinary oriented matroid theory, these observations on duality must be connected with a total vector space $R^{d+1}$ corresponding to $T_{\xi}(\mathcal{E})$, a subspace $L \subseteq R^{d+1}$ corresponding to $H_{\xi}(\mathcal{E})$ and the orthogonal complement $L^{\perp}$ corresponding to $V_{\xi}(\mathcal{E})$. Thus, just as $\left(H_{\xi}(\mathcal{E})\right.$, $\left.V_{\xi}(\mathcal{E})\right)$ determine the structure of $T_{\xi}(\mathcal{E})$, the dual pair $\left(L, L^{\perp}\right)$ determines the structure of total space $R^{d+1}$. Therefore, it may result very convenient to be able to introduce the concept of an oriented matroid in terms of the 
structure $\left(L, L^{\perp}\right)$ rather than only in terms of the subspace $L$. If this is possible then one should be able to make a direct transition

$$
\left(L, L^{\perp}\right) \longrightarrow\left(H_{\xi}(\mathcal{E}), V_{\xi}(\mathcal{E})\right),
$$

and in this way to ensure from the beginning the duality symmetry. Surprisingly, the mathematicians have already considered an equivalent definition of an oriented matroid in terms of an analog structure to the pair $\left(L, L^{\perp}\right)$. Such a definition used the concept of Farkas property, which we shall proceed now to discuss briefly (see ref. [19] for details).

But before we address the Farkas property (Lemma) it turns out necessary to introduce the sign vector concept. Let $E \neq \varnothing$ be a finite set. An element $X \in\{+,-, 0\}^{E}$ is called a sign vector. The positive, negative and zero parts of $X$ are denoted by $X^{+}, X^{-}$and $X^{0}$, respectively. Further, we define $\operatorname{supp} X \equiv X^{+} \cup X^{-}$. Consider two sets $S$ and $S^{\prime}$ of signed vectors. The pair $\left(S, S^{\prime}\right)$ is said to have the Farkas property, if $\forall e \in E$ either

(a) $\exists X \in S, e \in \operatorname{supp} X$ and $X \geq 0$

or

(b) $\exists Y \in S^{\prime}, e \in \operatorname{supp} Y$ and $Y \geq 0$

but not both. Here, $X \geq 0$ means that $X$ has a positive $(+)$ or a zero (0) entry in every coordinate. Observe that $\left(S, S^{\prime}\right)$ has the Farkas property if and only if $\left(S^{\prime}, S\right)$ has it.

Let $S$ be a set of signed vectors, and let $I$ and $J$ denote disjoint subsets of $E$. Then

$$
S \backslash I / J=\left\{\tilde{X} \mid \exists X \in S, X_{I}=0, X_{J}=*, X=\tilde{X} \quad \text { on } E \backslash(I \cup J)\right\}
$$

is called a minor of $S$ (obtained by deleting $I$ and contracting $J$ ). Here, the symbol "*" denotes and arbitrary value. If $S$ and $S^{\prime}$ are sets of sign vectors on $E$, then $\left(S \backslash I / J, S^{\prime} \backslash J / I\right)$ is called minor of $\left(S, S^{\prime}\right)$. Similarly,

$$
{ }_{I} S=\left\{\tilde{X} \mid \exists X \in S, X_{I}=-\tilde{X}_{I}, X_{E \backslash I}=\tilde{X}_{E \backslash I}\right\}
$$

is called the reorientation of $S$ on $I$. Further, $\left({ }_{I} S,{ }_{I} S^{\prime}\right)$ is the reorientation of $\left(S, S^{\prime}\right)$ on $I$. Moreover, $S$ is symmetric if $S=-S$, where $-S$ is the set of signed vectors which are opposite to the signed vectors of $S$.

With these definitions at hand, we are ready to give an alternative but equivalent definition of an oriented matroid. Let $E \neq \varnothing$ be a finite set and let $S$ and $S^{\prime}$ be two sets of sign vectors. Then the pair $\left(S, S^{\prime}\right)$ is called an oriented matroid on $E$, if [19]:

$(F 1) S$ and $S^{\prime}$ are symmetric and

$(F 2)$ every reorientation of every minor of $\left(S, S^{\prime}\right)$ has the Farkas property. 
From this definition follows that $\left(S, S^{\prime}\right)$ is an oriented matroid if and only if $\left(S^{\prime}, S\right)$ is an oriented matroid, and also that every minor and every reorientation of an oriented matroid is an oriented matroid again.

Two sign vectors $X$ and $Y$ are orthogonal, denoted by $X \perp Y$, if

$$
\left(X^{+} \cap Y^{+}\right) \cup\left(X^{-} \cap Y^{-}\right) \neq 0 \Longleftrightarrow\left(X^{+} \cap Y^{-}\right) \cup\left(X^{-} \cap Y^{+}\right) .
$$

The set

$$
S^{\perp}=\{X \mid X \perp S\},
$$

where $X \perp S$ means that $X \perp Y$ for every $Y \in S$, is called the orthogonal complement of $S$. If $S^{\prime} \subseteq S^{\perp}$ then we say that $S$ and $S^{\prime}$ are orthogonal. If $\left(S, S^{\prime}\right)$ is an oriented matroid then two important results follow, namely $S$ and $S^{\prime}$ are orthogonal and $\left(S, S^{\perp}\right)$ is also an oriented matroid.

A collection $\mathcal{C}$ of $\operatorname{sign}$ vectors on the set $E$ is the set of signed circuits of an oriented matroid on $E$ if and only if satisfies the following axioms:

$(C 0) \mathcal{C} \neq \varnothing$,

$(C 1) \mathcal{C}$ is symmetric,

(C2) for all $X, Y \in \mathcal{C}$, if $X \subseteq Y$, then $X=Y$ or $X=-Y$,

(C3) for all $X, Y \in \mathcal{C}, X \neq-Y$ and $e \in X^{+} \cap Y^{-}$, there is a $Z$ such that $e \neq \operatorname{supp} Z, Z^{+} \subseteq X^{+} \cup Y^{+}$and $Z^{-} \subseteq X^{-} \cup Y^{-}$.

Now, it can be proved that if $\left(S, S^{\prime}\right)$ is an oriented matroid then $S$ satisfies the axioms $(C 0)-(C 3)$. Indeed, it can be proved that the axioms $(F 1)-(F 2)$ and $(C 0)-(C 3)$ provide equivalent definitions of an oriented matroid (see ref. [19]).

The key link between the definition of an oriented matroid in terms of a chirotope $(\chi 1)-(\chi 3)$ and circuits $(C 0)-(C 3)$ is provided by the relation

$$
\mathcal{C}\left(x_{i}\right)=(-1)^{i} \chi\left(x_{1}, \ldots, x_{i-1}, x_{i+1}, \ldots, x_{r+1}\right),
$$

where $\left\{x_{1}, \ldots, x_{r}\right\}$ is a basis of $\mathcal{M}$ and

$$
\mathcal{C} \subset\left\{x_{1}, \ldots, x_{r}, x_{r+1}\right\} .
$$

Thus, although it is not straightforward to prove that the definitions $(\chi 1)-(\chi 3)$ and $(C 0)-(C 3)$ are equivalent, the relation $(24)$ gives an idea of the key step in such a proof. Consequently, we may conclude that $(\chi 1)-$ $(\chi 3),(C 0)-(C 3)$ and $(F 1)-(F 2)$ are three equivalent definitions of the same structure: an oriented matroid. 
Now, assume that $\left(S, S^{\perp}\right)$ is an oriented matroid. One question that arises is: can $\left(S, S^{\perp}\right)$ be realizable in terms of the pair $\left(L, L^{\perp}\right)$ of dual vector spaces, where $L, L^{\perp} \subseteq R^{d+1}$ ? The answer is, of course not always. This means that the oriented matroid concept is more general than the concept of a vector space. In fact, one can prove that a dual pair of realizable oriented matroids on $E$ corresponds to a pair of orthogonal subspaces in $R^{d+1}$. Moreover, one may also ask a related question: Is it possible to connect $\left(L, L^{\perp}\right)$ with a fiber bundle structure $\mathcal{E}(B, F, G)$ ? Matroid bundle seems to give an affirmative answer to this question. In fact, the main idea of a matroid bundle is to replace the fiber $F$ by an oriented matroid $\mathcal{M}\left(\xi^{a}\right)$, where $\xi^{a}$ are local coordinates on the base manifold $B$. However, this prescription is focused more on the definitions $(\chi 1)-(\chi 3)$ and $(C 0)-$ $(C 3)$ of an oriented matroid rather than on the definition $(F 1)-(F 2)$. But considering the equivalence between the three definitions $(\chi 1)-(\chi 3),(C 0)-$ $(C 3)$ and $(F 1)-(F 2)$, one should expect a definition for a matroid bundle in terms of the Farkas structure $(F 1)-(F 2)$. Our conjecture is that the tangent bundle $T(\mathcal{E})$ would be the central tool to achieve this goal. The reason is that $T(\mathcal{E})$ naturally admits the splitting

$$
T_{\xi}(\mathcal{E})=H_{\xi}(\mathcal{E}) \oplus V_{\xi}(\mathcal{E}),
$$

in terms of the horizontal $H_{\xi}(\mathcal{E})$ and vertical $V_{\xi}(\mathcal{E})$ subspaces of $T(\mathcal{E})$. In fact, one may think that the object $\left(H_{\xi}(\mathcal{E}), V_{\xi}(\mathcal{E})\right)$ constitutes a local version of the pair of complementary subspaces $\left(L, L^{\perp}\right)$. Therefore, just as the matroid bundle concept considers the transition $\mathcal{M} \rightarrow \mathcal{M}\left(\xi^{a}\right)$, based on any of the definitions $(\chi 1)-(\chi 3)$ and $(C 0)-(C 3)$, one may assume that the equivalent definition for a matroid bundle in terms of the definition $(F 1)$ $(F 2)$ should provide the transition $\left(L, L^{\perp}\right) \rightarrow\left(H_{\xi}(\mathcal{E}), V_{\xi}(\mathcal{E})\right)$. For physicists, this kind of scenario is not completely new since in Kaluza-Klein theory through the so-called spontaneous compactification mechanism, the vector space $R^{d+1}$ (or a manifold) is locally considered as a fiber bundle $B \times F$ and as a consequence the tangent bundle $T_{\xi}(\mathcal{E})$ splits as in $(26)$.

What could the connection between the matroid bundle theory in terms of the pair $\left(S, S^{\perp}\right)$ and $M$-theory be? Let us assume that the starting point in $M$-theory is a duality principle based on the dual symmetry contained in the Farkas property $(F 1)-(F 2)$, then one should expect that the pair $\left(S, S^{\perp}\right)$ plays a basic role in the partition function associated with any proposed $M$-theory, which we symbolically write as

$$
Z_{\left(S, S^{\perp}\right)}=\int D X \exp \left(S_{\left(S, S^{\perp}\right)}\right)
$$

As a consequence, due to the Farkas property one ensures a duality symmetry in (27) not only at the level of the full space $\left(S, S^{\perp}\right)$, but also for any subspace $\left(S \backslash I / J, S^{\prime} \backslash J / I\right)$ corresponding to any reoriented minor of $\left(S, S^{\perp}\right)$. 
In other words, the partition function

$$
Z_{\left(S \backslash I / J, S^{\prime} \backslash J / I\right)}=\int D X \exp \left(S_{\left(S \backslash I / J, S^{\prime} \backslash J / I\right)}\right)
$$

must also contain the dual symmetry. This suggests a split of the full partition function $Z_{\left(S, S^{\perp}\right)}$ in terms of fundamental minors $Z_{\left(S \backslash I / J, S^{\prime} \backslash J / I\right)}$. In fact, this conclusion is closely related to the oriented matroid result

$$
\left(\mathcal{M}_{1} \oplus \mathcal{M}_{2}\right)^{*}=\mathcal{M}_{1}^{*} \oplus \mathcal{M}_{2}^{*}
$$

where $\mathcal{M}_{1} \oplus \mathcal{M}_{2}$ is the direct sum of two oriented matroids $\mathcal{M}_{1}$ and $\mathcal{M}_{2}$ and $\mathcal{M}^{*}$ denotes the dual of $\mathcal{M}$.

In the case of $p$-branes physics one may think as follows. Let us associate the $p_{1}$-brane and $p_{2}$-brane with the matroids $\mathcal{M}_{1}$ and $\mathcal{M}_{2}$ respectively, then the corresponding partition functions

$$
Z_{\mathcal{M}_{1}}=\int D X \exp \left(S_{p_{1}}\right)
$$

and

$$
Z_{\mathcal{M}_{2}}=\int D X \exp \left(S_{p_{2}}\right)
$$

should lead to the dual symmetry $Z=Z^{*}$ of the total partition function $Z=Z_{\mathcal{M}_{2}} Z_{\mathcal{M}_{2}}$. Here, the actions $S_{p_{1}}$ and $S_{p_{2}}$ are determined by (9). Due to the equivalence between $(\chi 1)-(\chi 3)$ and $(F 1)-(F 2)$, one should expect that the similar conclusion must be true using an action for $p$-branes based on the Farkas property, which we symbolically write as $S_{\left(p, p^{\perp}\right)}$, where $p^{\perp}=$ $d-p-1$. But once again a question emerges: what could the form of $S_{\left(p, p^{\perp}\right)}$ be? This action should contain the invariance $S_{\left(p, p^{\perp}\right)}=S_{\left(p^{\perp}, p\right)}$ and for that reason one should expect that the action $S_{\left(p, p^{\perp}\right)}$ would combine the objects $\sigma^{\mu_{1} \cdots \mu_{p+1}}$ and ${ }^{*} \sigma^{\mu_{p+2} \cdots \mu_{d+1}}$, which are related according to (15). In analogy to (9), one discovers that the simplest possibility seems to be

$$
\begin{aligned}
& S_{\left(p, p^{\perp}\right)} \\
& \quad=\frac{1}{2(d+1) !} \int d^{d+1} \xi\left(\gamma^{-1} \varepsilon^{\mu_{1} \cdots \mu_{p+1} \mu_{p+2} \cdots \mu_{d+1}} \sigma_{\mu_{1} \cdots \mu_{p+1}}{ }^{*} \sigma_{\mu_{p+2} \cdots \mu_{d+1}}-\gamma T_{p}^{2}\right) .
\end{aligned}
$$

We observe that the integrand in this action is over the whole fiber bundle space $\mathcal{E}$. The reason for this is that according to (10) and (16) $\sigma^{\mu_{1} \cdots \mu_{p+1}}$ is defined over the horizontal space $H_{\xi}(\mathcal{E})$ whereas ${ }^{*} \sigma_{\mu_{p+2} \cdots \mu_{d+1}}$ is defined over the vertical space $V_{\xi}(\mathcal{E})$, and therefore the integrand in (32) must be over the dimension of the tangent bundle $T_{\xi}(\mathcal{E})$, which is equal to $d+1$. 
Using (15), it is not difficult to see that the action $S_{\left(p, p^{\perp}\right)}$ can be written in any of the alternative ways:

$$
S_{(p, 0)}=\frac{1}{2(d+1) !} \int d^{d+1} \xi\left(\gamma^{-1} \sigma^{\mu_{1} \cdots \mu_{p+1}} \sigma_{\mu_{1} \cdots \mu_{p+1}}-\gamma T_{p}^{2}\right)
$$

or

$$
S_{\left(0, p^{\perp}\right)}=\frac{1}{2(d+1) !} \int d^{d+1} \xi\left(\gamma^{-1 *} \sigma^{\mu_{p+2} \cdots \mu_{d+1}}{ }^{*} \sigma_{\mu_{p+2} \cdots \mu_{d+1}}-\gamma T_{p}^{2}\right) .
$$

We observe that in general we have $x^{\mu}\left(\xi^{a}, \xi^{\hat{a}}\right)$. Therefore, in order to derive the action (9) from (33) we need to assume $x^{\mu}\left(\xi^{a}, \xi^{\hat{a}}\right)=f\left(\xi^{\hat{a}}\right) x^{\mu}\left(\xi^{a}\right)$, so that we can integrate out the coordinates $\xi^{\hat{a}}$. We observe, however, that in this case the action $S_{\left(0, p^{\perp}\right)}$ vanishes. Similarly, if we assume that $x^{\mu}\left(\xi^{a}, \xi^{\hat{a}}\right)=$ $g\left(\xi^{a}\right) x^{\mu}\left(\xi^{\hat{a}}\right)$ then action (32) leads to a $p^{\perp}$-brane action, but the $p$-brane action $S_{(p, 0)}$ vanishes. This means that the action (32) is more general than the actions (33) and (34). It is interesting to observe that in the case $d+1=2 n$ the action (32) can be reduced to the Zaikov self-dual action [20], which has been studied in some detail by Castro [21].

In order to clarify the meaning of (32), let us assume that the action $S_{p}$ corresponds to a 1-brane, with $d+1=8$. In this case the dual action $S_{p \perp}$ corresponds to a 5 -brane action. But, the interesting aspect of (32) is that $S_{\left(p, p^{\perp}\right)}$ combines both the 1-brane and 5-brane in a unified way. Thus, the corresponding partition function

$$
Z_{\left(S, S^{\perp}\right)}=\int D X \exp \left(S_{(1,5)}\left(S, S^{\perp}\right)\right)
$$

should describe the quantum dynamics of both the 1-brane and 5-brane.

Let us make some final comments. Just as duality is a concept of fundamental importance in $M$-theory the same is true for oriented matroid theory. In fact, all objects defined in oriented matroid theory can be dualized. In particular since $\mathcal{M}^{* *}=\mathcal{M}$, an oriented matroid $\mathcal{M}$ can be defined either directly or dually. Due to this duality features of oriented matroid theory, perhaps a more appropriate name for matroid theory should be 'duality theory'. In view of the duality definition (12)-(14), in terms of chirotopes, it becomes evident that oriented matroid may provide the mathematical framework for implementing a duality principle in $M$-theory. This conjecture becomes more evident using the Farkas definition of an oriented matroid. This suggests a partition function for $M$-theory which automatically ensures the duality symmetry not only for the dual space $\left(S, S^{\perp}\right)$ but for any minor of $\left(S, S^{\perp}\right)$ as well. These observations lead us to discover the action (32) which dually combines both the $p$-brane and its dual $p^{\perp}$-brane. 
Yet one may wonder whether the action (32) is unique. As we mentioned just below expression (14), every oriented matroid $\mathcal{M}(E, \chi)$ has an associated unique dual $\mathcal{M}^{*}\left(E, \chi^{*}\right)$ and $\mathcal{M}^{* *}=\mathcal{M}$ (see ref. [6], Section 3.4). This implies that starting with $\sigma_{\mu_{1} \cdots \mu_{p+1}}$ we find that the dual chirotope ${ }^{*} \sigma_{\mu_{p+2} \cdots \mu_{d+1}}$ is unique and vice versa starting with ${ }^{*} \sigma_{\mu_{p+2} \cdots \mu_{d+1}}$ we discover that the chirotopes $\sigma_{\mu_{1} \cdots \mu_{p+1}}$ is unique. Thus, the action (32) is constructed with two unique chirotopes $\sigma_{\mu_{1} \cdots \mu_{p+1}}$ and ${ }^{*} \sigma_{\mu_{p+2} \cdots \mu_{d+1}}$. It remains to be seen whether the combination of $\sigma_{\mu_{1} \cdots \mu_{p+1}}$ and ${ }^{*} \sigma_{\mu_{p+2} \cdots \mu_{d+1}}$ given in (32) is unique. First observe that the indices of the combination $\sigma_{\mu_{1} \cdots \mu_{p+1}}{ }^{*} \sigma_{\mu_{p+2} \cdots \mu_{d+1}}$ go from $\mu_{1}$ to $\mu_{d+1}$. This means that one requires a completely antisymmetric object with $d+1$ indices. Thus, one can associate such an object with a chirotope with $d+1$ indices. Considering a ground set of the form $E_{d+1}=$ $\{1, \ldots, d+1\}$, one discovers that the object $\varepsilon^{\mu_{1} \cdots \mu_{p+1} \mu_{p+2} \cdots \mu_{d+1}}$ is the required chirotope which is unique (see ref. [1]).

\section{Acknowledgments}

I would like to thank E. Sezgin and Texas A\&M University Physics Department for their kind hospitality, where part of this work was developed.

\section{References}

[1] J.A. Nieto, Adv. Theor. Math. Phys. 8 (2004), 177, hep-th/0310071.

[2] J.A. Nieto, J. Math. Phys. 45 (2004), 285, hep-th/0212100.

[3] J.A. Nieto and M.C. Marín, Int. J. Mod. Phys. A 18 (2003), 5261, hep-th/0302193.

[4] J.A. Nieto and M.C. Marín, J. Math. Phys. 41 (2000), 7997.

[5] J.A. Nieto, Rev. Mex. Fis. 44 (1998), 358.

[6] A. Björner, M. Las Vergnas, B. Sturmfels, N. White and G.M. Ziegler, Oriented martroids, Cambridge University Press, Cambridge, 1993.

[7] P.K. Townsend, Four lectures on M-theory, Proceedings of the ICTP on the Summer School on High Energy Physics and Cosmology, June 1996, hep-th/9612121.

[8] M.J. Duff, Int. J. Mod. Phys. A 11 (1996), 5623, hep-th/9608117.

[9] P. Horava and E. Witten, Nucl. Phys. B 460 (1996), 506.

[10] E.J. Martinec, Matrix theory and $N=(2,1)$ strings, hep-th/9706194.

[11] M.J. Duff and J.X. Lu, Classical Quant. Grav. 9 (1992), 1. 
[12] R.D. MacPherson, Combinatorial differential manifolds: a symposium in honor of John Milnor's sixtieth birthday, in Topological Methods on Modern Mathematics (Stony Brook, NY, 1991), eds. L.H. Goldberg and A. Phillips, Houston, 1993, 203-221.

[13] L. Anderson and J.F. Davis, Mod 2 cohomolgy of combinatorial Grassmannians, Selecta Math. 8 (2002), 161; math.GT/9911158.

[14] L. Anderson, New Perspectives Geom. Comb. 38 (1999), 1.

[15] D. Biss, Some applications of oriented matroids to topology, Ph.D. Thesis, MIT, 2002.

[16] A. Schild, Phys. Rev. D 16 (1977), 1722.

[17] J. Gamboa, C. Ramírez and M. Ruiz-Altaba, Nucl. Phys. B 338 (1990), 143.

[18] J.G. Oxley, Martroid theory, Oxford University Press, New York, 1992.

[19] A. Bachem and W. Kern, Linear programming duality, Spring-Verlag, Berlin, Heidelberg, 1992.

[20] R.P. Zaikov, Mod. Phys. Lett. A 5 (1990), 911.

[21] C. Castro, The Selfdual Supermembrane and the SuperToda, molecule, Phys. Lett. B 288 (1992), 291. 
